\title{
Noise reduction for enzymatic reactions: a case study for stochastic product clearance
}

\author{
A. Borri P. Palumbo A. Singh
}

\begin{abstract}
A basic (though rather general) enzymatic reaction scheme is investigated here, with a substrate that transforms into a product by means of the catalytic action of an enzyme. The aim is of quantifying the effects of feedback in noise propagation. Noise sources are twofold: one affects the enzyme production, assuming to happen according to finite bursts of molecules; the other concerns the product clearance, with the classical linear elimination rate affected by a Bernoulli random variable that can switch 'on' or 'off' the clearance. Two distinct feedback control schemes on enzyme production are considered here: one from the final product of the pathway activity, the other from the enzyme accumulation (negative autoregulation). Metabolic noise is defined in terms of the square of the coefficient of variation of the product, and computations are carried out by means of moment equations. Results show that, according to the type of the feedback parameter chosen to tune the feedback action, one of the two feedback schemes is preferable to the other with respect to noise reduction.
\end{abstract}

Index Terms-Enzymatic reactions, Moment Equations, Negative Feedback

\section{INTRODUCTION}

Mathematical control theory has recently gained more and more interest in the synthetic biology community, aiming at merging molecular biological techniques with mathematical modeling and forward engineering in order to design synthetic biological circuits, able to replicate emergent properties potentially useful for biotechnology industry, human health and environment (see [1], [2], [3], [4] and references therein). In this framework, recent attention has been focused on understanding how circuit design may affect metabolic performances, and a pivotal role seems to be played by feedback mechanisms regulating the enzymatic activity. The role of the feedback in systems and synthetic biology has been widely investigated, especially in transcriptional and metabolic regulation where gene products are required to control their homeostatic levels robustly with respect to parameter or environmental fluctuations [5], [6], [7], [8], [9], [10], [11], [12], [13].

The framework investigated here is that of a basic (though rather general) metabolic pathway, involving the classical substrate/enzyme binding/unbinding forming a complex that eventually provides a final product (with the release of the

A. Borri, and P. Palumbo are with the Istituto di Analisi dei Sistemi e Informatica "A. Ruberti", Italian National Research Council (IASI-CNR), Viale Manzoni, Roma, Italy. Email addresses: \{alessandro.borri, pasquale.palumbo\}eiasi.cnr.it

A. Singh is with Department of Electrical and Computer Engineering, Biomedical Engineering, Mathematical Sciences, Center for Bioinformatics and Computational Biology, University of Delaware, Newark, DE USA 19716. E-Mail address: absingh@udel. edu enzyme). Differently from closed frameworks where total substrate and enzyme are conserved, here productions and clearance processes are considered. Two sources of noise are envisioned. One affects the enzyme production and is modeled by means of finite bursts of molecules, the amount of which follows a geometric distribution; the other source of noise affects the product elimination, which can be active (or inactive) according to the state equal to 1 (or equal to 0 ) of a Bernoulli random variable [14], [15], [16]. The aim is to evaluate the effect of a feedback in the enzymatic production rate on the product noise propagation. The feedback on the enzyme may be exerted via a transcriptional repression from the product or from the enzyme itself.

Moment equations are exploited to compute the metabolic noise in terms of the square of the coefficient of variation of the product around the steady-state solution. To this end a Stochastic Hybrid System (SHS) model is adopted with the deterministic part of the SHS made of Reaction Rate Equations (RRE) and the only two sources of stochasticity accounted for the bursty enzyme production and product elimination. Similarly to [11], substrate is not supposed to vary, thus accounting for scenarios in which the substrate is an extracellular nutrient pool consumed by a low-density cell population. Such an assumption excludes complete depletion of the substrate, eventually providing a nil equilibrium. A similar study has been proposed in [11] where enzyme production was not modeled by bursts and in [12], [13], where substrate was allowed to vary. A further difference with respect to [11] and [12], [13] is the presence of a noisy product elimination.

Despite the presence of a second order dynamics for the product elimination, moment equations can be written in closed form, because of the choice of the Bernoulli variable. Computations are carried out by properly exploiting the Quasi Steady-State Approximation, a widespread approach employed to reduce the computational complexity in the presence of a typical fast/slow time-scale of enzymatic reactions: see e.g. [17], [18] and references therein for an exhaustive review of advantages and limitations of such approach, which substantially exploits the faster dynamics of the complex, supposed to be negligible with respect to the other players' dynamics.

Similarly to [11], solutions focus on a pair of feedback parameters (promoter and repression strength) and suggest which of the two feedback schemes should be preferred to the other in terms of a greater sensitivity to the chosen feedback parameter and of a better improvement in noise reduction. Analytical solutions for the scheme involving the feedback from the enzyme are as well provided. 


\section{Chemical Reaction Schemes}

The chemical reaction schemes under investigation are reported in Figs. 1 and 2.

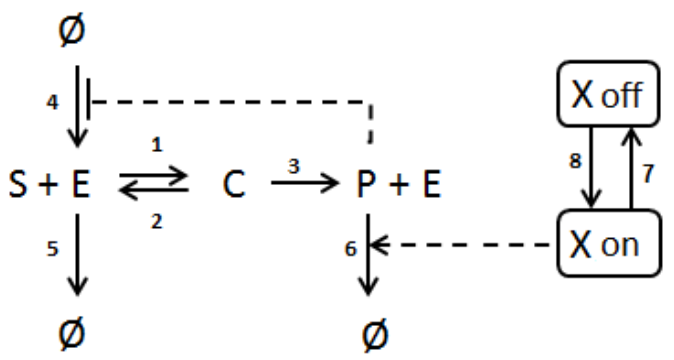

Fig. 1. Metabolic reaction framework: feedback from the product $P$

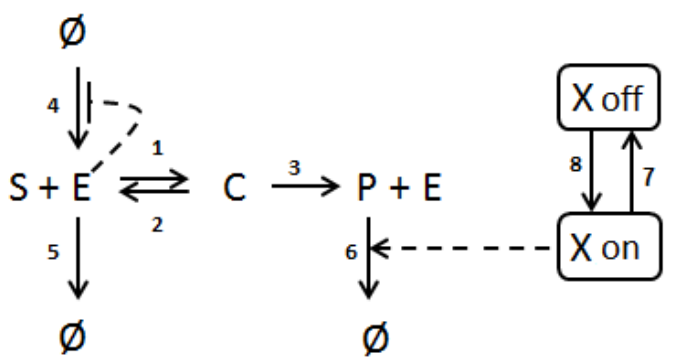

Fig. 2. Metabolic reaction framework: feedback from the enzyme $E$

The common part consists of a substrate $S$ binding to an enzyme $E$ in order to form a complex $C$ (reaction 1), which in turn can reverse the binding (reaction 2) or can be transformed into a product $P$ with the release of the enzyme $E$ (reaction 3). The reaction schemes account for enzyme production (reaction 4), enzyme degradation (reaction 5) and product elimination (for instance due to its final utilization, reaction 6). The substrate is not supposed to vary.

Product clearance is supposed to be regulated by a stochastic switch, modeled by a Bernoulli random variable $X$. If $X=1$ the product is eliminated according to a typical linear clearance rate; if $X=0$ product elimination is inhibited. The switch of $X$ is independent of the other random processes. The use of a Bernoulli random variable assuming values in $\{0,1\}$ allows to reduce moment computations because moments involving higher order powers of $X$ trivially reduce as follows

$$
\left\langle X^{k}\right\rangle=\langle X\rangle, \quad\left\langle Y^{j} X^{k}\right\rangle=\left\langle Y^{j} X\right\rangle,
$$

for any other random variable $Y$ and any integers $j, k$.

Enzyme production is affected by a second noise source, since it occurs in bursts of $B_{e}$ copy numbers, with the random variable $B_{e}$ indicating the size (in terms of number of copies) of the bursts, occurring with probabilities $\mathbb{P}\left(B_{e}=j\right)$ with $j \in\{0,1, \ldots\}$. As in [15], [19] we assume a geometric probability distribution:

$$
\mathbb{P}\left(B_{e}=j\right)=(1-\lambda)^{j} \lambda, \quad \lambda \in(0,1], \quad j=0,1, \ldots
$$

TABLE I

TRANSITIONS AND ASSOCIATED PROPENSITIES FOR THE DISCRETE STOCHASTIC EVENTS

\begin{tabular}{|c|c|c|}
\hline Event & Reset & Propensities \\
\hline \hline $\begin{array}{c}\text { Enzyme production } \\
\text { non-feedback case }\end{array}$ & $n_{e} \mapsto n_{e}+j, j=0,1, \ldots$ & $k_{4} \mathbb{P}\left(B_{e}=j\right)$ \\
\hline $\begin{array}{c}\text { Enzyme production } \\
\text { feedback from } P\end{array}$ & $n_{e} \mapsto n_{e}+j, j=0,1, \ldots$ & $f_{p}\left(n_{p}\right) \mathbb{P}\left(B_{e}=j\right)$ \\
\hline $\begin{array}{c}\text { Enzyme production } \\
\text { feedback from } E\end{array}$ & $n_{e} \mapsto n_{e}+j, j=0,1, \ldots$ & $f_{e}\left(n_{e}\right) \mathbb{P}\left(B_{e}=j\right)$ \\
\hline$X$ activation & $X \mapsto X+1$ & $k_{8}(1-X)$ \\
\hline$X$ deactivation & $X \mapsto X-1$ & $k_{7} X$ \\
\hline
\end{tabular}

providing an average burst size $\left\langle B_{e}\right\rangle=(1-\lambda) / \lambda$.

Two kinds of feedbacks are considered, both working on the enzyme production: one from the product $P$ (scheme in Fig.1), the other from the enzyme $E$ itself (scheme in Fig.2). According to the feedback schemes, the propensities associated to the noisy bursts are proportional to the following sigmoidal Hill functions, [11]:

$$
f_{i}\left(n_{i}\right)=\frac{k_{4}}{1+\left(n_{i} / \theta_{i}\right)^{h_{i}}}, \quad i=p, e .
$$

with $n_{p}, n_{e}$ denoting the product and enzyme copy number. Parameter $k_{4}$ is the promoter strength, providing the maximal propensity $k_{4} \mathbb{P}\left(B_{e}=j\right)$ associated to the production of $B_{e}=j$ copy number of enzyme, obtainable for negligible values of the entries $n_{p}, n_{e}$ : when $n_{i} \ll \theta_{i}$ the two feedback schemes collapse to the not-regulated case. Half of the maximal value of the propensity is reached in correspondence of the repression thresholds, $\theta_{i}$. Parameters $h_{i}$ are the promoter sensitivities, providing the steepness of the sigmoidal functions.

Discrete stochastic events and associated propensities are resumed in Table I.

\section{A. Metabolic noise computation}

Referring to the product $P$, we define the corresponding metabolic noise by means of the square of the coefficient of variation $C V_{P}^{2}$ computed by the ratio:

$$
\eta_{P, i}^{2}=\sigma_{P}^{2} /\left(n_{p}^{\star}\right)^{2}, \quad i=p, e
$$

where $\sigma_{P}^{2}$ and $n_{p}^{\star}$ are the steady-state values for variance and mean of the marginal distribution of the product $P$ copy number. The suffix $i$ indicates the case of the feedback from the product $(i=p)$ or from the enzyme $(i=e)$. Since by varying the feedback parameters, the steady-state average solutions vary as well, similarly to [11], comparison is made with respect to the relative noise

$$
\tilde{\eta}_{P, i}^{2}=\eta_{P, i}^{2} / \eta_{P}^{2}
$$

with $\eta_{P}^{2}$ denoting the metabolic noise for the non-feedback case.

In order to evaluate the metabolic noise, we consider a Stochastic Hybris Systems (SHS) modeling framework, where a Reaction Rate Equation (RRE) system is associated to the reaction schemes evolving between any two discrete 
stochastic events given by burst enzyme production or $X$ switch (Table I). By identifying the state of the system by the copy number of each involved species $n_{e}(t), n_{c}(t), n_{p}(t)$, plus the state of $X$ regulating the product elimination $(X=1$ for active and $X=0$ for non-active elimination of $P$ ), the RRE is:

$$
\begin{aligned}
& \dot{n}_{e}(t)=-k_{1} n_{s} n_{e}(t)+\left(k_{2}+k_{3}\right) n_{c}(t)-k_{5} n_{e}(t) \\
& \dot{n}_{c}(t)=k_{1} n_{s} n_{e}(t)-\left(k_{2}+k_{3}\right) n_{c}(t) \\
& \dot{n}_{p}(t)=k_{3} n_{c}(t)-k_{6} X(t) n_{p}(t)
\end{aligned}
$$

According to our assumptions, $n_{s}$ is the substrate copy number and is kept constant.

In the following, unless differently specified, the expected value of a random variable $x$ is denoted by $\langle x\rangle$, while the steady-state of the average of a stochastic process $x(t)$ or of a second order moment $\langle x(t) y(t)\rangle$ are denoted by $x^{\star}=\lim _{t \mapsto+\infty}\langle x(t)\rangle$ and $\langle x y\rangle^{\star}=\lim _{t \mapsto+\infty}\langle x(t) y(t)\rangle$, respectively.

\section{Average Steady-STATE SOlutions}

The first-order moment equations associated to the SHS modeling the reaction schemes are derived from [20]. Unfortunately, the nonlinearities involved in the enzyme production for the feedback schemes do not allow to achieve closedform solutions. Indeed, the nonlinear terms provided by the negative feedback schemes even prevent to use the moment closure techniques [21]. Therefore, computations are carried out according to the linearization of the nonlinear propensities around the stationary average values $n_{e}^{\star}, n_{p}^{\star}$ :

$$
\begin{aligned}
& f_{p}\left(n_{p}(t)\right) \simeq f_{p}\left(n_{p}^{\star}\right)+f_{p}^{\prime}\left(n_{p}^{\star}\right)\left(n_{p}(t)-n_{p}^{\star}\right) \\
& f_{e}\left(n_{e}(t)\right) \simeq f_{e}\left(n_{e}^{\star}\right)+f_{e}^{\prime}\left(n_{e}^{\star}\right)\left(n_{e}(t)-n_{e}^{\star}\right) .
\end{aligned}
$$

Clearly, such an approximation is valid as long as the stochastic fluctuations do not leave the region in which the Hill function is approximately linear.

According to [20], the first order moment equations can be written in a unified fashion for the two feedback schemes, with the steady-state solutions obeying to the following system:

$$
\begin{aligned}
& -k_{1} n_{s} n_{e}^{\star}+\left(k_{2}+k_{3}\right) n_{c}^{\star}-k_{5} n_{e}^{\star}+\chi\left(n_{p}^{\star}, n_{e}^{\star}\right)=0 \\
& k_{1} n_{s} n_{e}^{\star}-\left(k_{2}+k_{3}\right) n_{c}^{\star}=0 \\
& k_{3} n_{c}^{\star}-k_{6}\left\langle X n_{p}\right\rangle^{\star}=0 \\
& k_{8}-\left(k_{7}+k_{8}\right) X^{\star}=0
\end{aligned}
$$

with

$$
\chi\left(n_{p}, n_{e}\right)= \begin{cases}k_{4}\left\langle B_{e}\right\rangle, & \text { non feedback case } \\ f_{p}\left(n_{p}\right)\left\langle B_{e}\right\rangle, & \text { feedback from } P, \\ f_{e}\left(n_{e}\right)\left\langle B_{e}\right\rangle, & \text { feedback from } E .\end{cases}
$$

Differently from previous frameworks [11], [12], [13] the presence of $X$ correlating to the other state variables prevents a trivial computation of the first order moments, except for

$$
X^{\star}=\frac{k_{8}}{k_{7}+k_{8}}
$$

Indeed, from standard computations, we have:

$$
\begin{gathered}
n_{e}^{\star}=\frac{\chi\left(n_{p}^{\star}, n_{e}^{\star}\right)}{k_{5}}, \quad n_{c}^{\star}=\frac{k_{1} n_{s}}{k_{2}+k_{3}} n_{e}^{\star}, \\
\left\langle X n_{p}\right\rangle^{\star}=\frac{k_{3} n_{c}^{\star}}{k_{6}}=\frac{k_{1} k_{3} n_{s}}{\left(k_{2}+k_{3}\right) k_{6}} n_{e}^{\star}
\end{gathered}
$$

In the following the constant parameter $k_{1} k_{3} n_{s} /\left(k_{2}+k_{3}\right)$ will be shortly denoted by $k_{s}$. It is apparent that even the non-feedback case (with $\chi\left(n_{p}^{\star}, n_{e}^{\star}\right)=k_{4}\left\langle B_{e}\right\rangle$ ) requires some of the second order moment equations for the computation of the average steady-states, due to the second order moment $\left\langle X n_{p}\right\rangle^{\star}$. Nevertheless, both feedback schemes allow to exactly compute in closed form the steady-state solutions. Indeed the computation of $\left\langle X n_{e}\right\rangle$ and $\left\langle X n_{p}\right\rangle$ dynamics provides at steady-state:

$$
\begin{gathered}
\quad \frac{d\left\langle X n_{e}\right\rangle}{d t}=0 \Longrightarrow\left\langle X n_{e}\right\rangle^{\star}=X^{\star} n_{e}^{\star} \\
\frac{d\left\langle X n_{p}\right\rangle}{d t}=0 \\
\Longrightarrow \quad k_{8} n_{p}^{\star}=\left(k_{6}+k_{7}+k_{8}\right)\left\langle X n_{p}\right\rangle^{\star}-k_{s}\left\langle X n_{e}\right\rangle^{\star} \\
=k_{s}\left(\frac{k_{6}+k_{7}+k_{8}}{k_{6}}-X^{\star}\right) n_{e}^{\star}=\frac{k_{s}\left(\left(1-X^{\star}\right) k_{6}+k_{7}+k_{8}\right)}{k_{6}} n_{e}^{\star}
\end{gathered}
$$

It worths noticing that (13)-(14) hold for both feedback schemes, with the steady-state average product $n_{p}^{\star}$ proportional to the steady-state average enzyme $n_{e}^{\star}$, if the switch parameters are kept constant. In summary, the first of (11) and (14) provide a unique solution for the pair $\left(n_{e}^{\star}, n_{p}^{\star}\right)$ according to which $n_{c}^{\star}$ is straightforwardly computed. Such a computation is trivial for the non-feedback case, since the two equations can be easily decoupled and solutions are analytical. On the other hand, in case of a feedback (from the enzyme or the product) we need to find numerically the solution.

\section{A. Qualitative behavior for varying parameters $k_{4}$ and $\theta_{p}$}

Qualitative analysis of first order steady-state solutions helps us to infer information on the feedback action. For instance, the following behavior is shared by both the feedback schemes:

i) feedback actions reduce the steady-state averages with respect to the non-feedback case; this is because, according (3), it is $k_{4}>f_{i}\left(n_{i}^{\star}\right)$ for any value of $n_{i}^{\star}$ and any $i=e, p$;

ii) by increasing the promoter strength $k_{4}$, then function $\chi\left(n_{p}^{\star}, n_{e}^{\star}\right)$ indefinitely increases, and so does $n_{e}^{\star}$ because of the first of (11) and, consequently, so does $n_{p}^{\star}$ because of (14); analogously, by reducing $k_{4} \mapsto 0^{+}, n_{e}^{\star}, n_{p}^{\star} \mapsto$ $0^{+}$;

iii) by increasing the feedback threshold both feedback schemes reduce to the constitutive case with $n_{e}^{\star}=$ $k_{4}\left\langle B_{e}\right\rangle / k_{5}$, whilst by reducing $\theta_{e}, \theta_{p} \mapsto 0^{+}$, the inhibitory action of the feedback becomes more and more effective, so that $n_{e}^{\star}, n_{p}^{\star} \mapsto 0$.

In case of a feedback from the product, when $n_{p}^{\star}=\theta_{p}$, then $f_{p}\left(n_{p}^{\star}=\theta_{p}\right)=k_{4} / 2$ whatever $h_{p}$. Such a position allows 
to immediately find $n_{e}^{\star}=k_{4}\left\langle B_{e}\right\rangle /\left(2 k_{5}\right)$ and, consequently from (14), it provides the following constraint among the parameters:

$$
k_{8} \theta_{p}=\frac{k_{4} k_{s}\left\langle B_{e}\right\rangle\left(\left(1-X^{\star}\right) k_{6}+k_{7}+k_{8}\right)}{2 k_{5} k_{6}}
$$

Analogously, in case of a feedback from the enzyme we have $f_{e}\left(n_{e}^{\star}=\theta_{e}\right)=k_{4} / 2$ whatever $h_{e}$. In this case, the straightforward constraint involves only the first of (11), providing $\theta_{e}=k_{4}\left\langle B_{e}\right\rangle /\left(2 k_{5}\right)$, according to which thresholds for $k_{4}$ (given $\theta_{e}$ ) or for $\theta_{e}$ (given $k_{4}$ ) are readily computed.

\section{B. Qualitative behavior for varying parameter $\left\langle B_{e}\right\rangle$}

If we investigate the role of the two noise sources in the steady-state solutions we find that the noisy bursts directly influence $n_{e}^{\star}$ and, as a matter of fact, also $n_{p}^{\star}$ : in both feedback schemes, as well as in the non-feedback case, the average burst size $\left\langle B_{e}\right\rangle$ plays the same role of the promoter strength $k_{4}$.

\section{Qualitative behavior for varying parameter $k_{8}$}

On the other hand, regards to the noise in the product elimination, in case of a feedback from the enzyme, $X$ does not affect $n_{e}^{\star}$. Instead, it clearly modifies $n_{p}^{\star}$. Indeed, according to (14), if we suppose to arbitrarily vary $k_{8}$ and, correspondingly, to vary $k_{7}$ in order to keep fixed $X^{\star}$, we have the following limits:

$$
\lim _{k_{8} \mapsto 0^{+}} n_{p}^{\star}=+\infty \quad \lim _{k_{8} \mapsto+\infty} n_{p}^{\star}=\frac{k_{s}}{k_{6} X^{\star}} n_{e}^{\star}
$$

Instead, regards to the feedback from the product, the stochastic switch $X$ affects both $n_{e}^{\star}$ and $n_{p}^{\star}$. In this feedback scheme, if we vary $k_{7}$ and $k_{8}$ in order to keep $X^{\star}$ fixed, we find that for lower and lower values of $k_{8}$ then $n_{e}^{\star}$ and $n_{p}^{\star}$ have an opposite behavior, the former definitely decreasing to 0 , the latter definitely increasing to $+\infty$. Indeed, when $k_{8} \mapsto 0^{+}$, equation (14) becomes the horizontal line $n_{e}=0$ in the $\left(n_{p}^{\star}, n_{e}^{\star}\right)$-phase space, intersecting the Hill function given by the first equation of (11) in $n_{p}^{\star}=+\infty$ and $n_{e}^{\star}=0$. On the other hand, for $k_{8} \mapsto+\infty$, equation (14) becomes $n_{e}^{\star}=\left(k_{6} / k_{s}\right) X^{\star} n_{p}^{\star}$ thus providing a finite, nontrivial solution for the pair $\left(n_{e}^{\star}, n_{p}^{\star}\right)$.

In summary, whatever is the chosen feedback scheme, a low frequency of switch $\left(k_{8} \mapsto 0^{+}\right)$, keeping fixed the average value $X^{\star}$, provides an increase of the steadystate average product (with a corresponding decrease of the steady-state average enzyme only according to the feedback from product); instead, a high frequency of switch $\left(k_{8} \mapsto\right.$ $+\infty$ ), keeping fixed $X^{\star}$, provides the asymptotic decrease of the steady-state average product to a limit point.

\section{SECOND ORDER MOMENTS}

Computations are carried out by properly applying the Quasi Steady-State Approximation (QSSA) to the RRE of the SHS, deterministically evolving between any two discrete events. The QSSA substantially exploits the faster dynamics of complex $C$, supposed to be negligible (i.e. $\dot{n}_{c}=0$ ) with respect to the other players' dynamics. By setting $\dot{n}_{c}=0$
TABLE II

Model Parameters. Measurement Units: $k_{1},\left[\mathrm{~S}^{-1}\right.$ MOLECUle $\left.^{-1}\right]$;

$$
k_{x}, x=2,3, \ldots, 7,8,\left[\mathrm{~s}^{-1}\right] \text {. }
$$

\begin{tabular}{|c|c|c|c|c|c|c|c|c|}
\hline Parameter & $k_{1}$ & $k_{2}$ & $k_{3}$ & $k_{5}$ & $k_{6}$ & $k_{7}$ & $k_{8}$ & $\lambda$ \\
\hline Value & 1 & 28300 & 3.2 & 0.02 & 0.02 & 0.3 & 0.9 & 0.25 \\
\hline
\end{tabular}

and substituting the expression of the complex $n_{c}$ in terms of the other two players (plus $X$ ), the RRE system reduces to:

$$
\begin{aligned}
& \dot{n}_{e}(t)=-k_{5} n_{e}(t) \\
& \dot{n}_{p}(t)=k_{s} n_{e}(t)-k_{6} X(t) n_{p}(t) .
\end{aligned}
$$

Clearly, the QSSA does not affect steady-state results achieved by (10)-(14).

Despite the presence of the nonlinear term in the product clearance rate, second-order moments can be written in a closed form without moment closure. This is because the nonlinearity involves a Bernoulli random variable assuming values in $\{0,1\}$, so that third order terms of the type $\left\langle X^{2} n_{i}\right\rangle$, $i=e, p$ can be reduced to second order terms $\left\langle X n_{i}\right\rangle$. In summary, accounting for the fact that first-order moments are achieved in (10)-(14) together with the second order moments $\left\langle X n_{e}\right\rangle^{\star},\left\langle X n_{p}\right\rangle^{\star}$, in order to compute $\left\langle n_{p}^{2}\right\rangle$ (necessary to estimate the relative noise $\tilde{\eta}_{P, i}^{2}, i=p, e$ ) the following second and third order moments are required: $\left\langle n_{e}^{2}\right\rangle,\left\langle n_{p}^{2}\right\rangle$, $\left\langle n_{e} n_{p}\right\rangle,\left\langle X n_{e}^{2}\right\rangle,\left\langle X n_{p}^{2}\right\rangle,\left\langle X n_{e} n_{p}\right\rangle$. By properly writing these moment equations, when looking for the steady-state solutions, because of the properties of $X$, we obtain a closed linear system of 6 unknowns.

\section{A. Feedback from the product}

Regards to the feedback from the product, analytical solutions are somehow cumbersome and are not reported here; instead exact solutions are readily numerically provided. Solutions for varying values of the promoter strength $k_{4}$ and of the repression strength $1 / \theta_{p}$ are reported in the upper panels of Figs.3 and 4, respectively, with not-varying parameter values reported in Table II.

It is apparent that low values of the promoter strength $k_{4}$ or of the repression strength $1 / \theta_{p}$ do not provide any effect of the feedback in terms of noise attenuation since the relative noise $\tilde{\eta}_{P, p}^{2} \simeq 1$. Such a framework is that described at the end of Subsection III-A. On the other hand, by increasing $k_{4}$ or $1 / \theta_{p}$ the relative noise varies. In both cases we have a non-monotonic behavior. Regards to the case of varying $k_{4}$, when the feedback sensitivity is $>1, \tilde{\eta}_{P, p}^{2}$ first decreases to a minimum, then increases to a maximum $>1$ and finally asymptotically approaches a steady-state value $<1$; instead, when $h_{p}=1$ the initial minimum is lost. On the other hand, when the repression strength increases we find first a minimum and, then, $\tilde{\eta}_{P, p}^{2}$ diverges to $+\infty$. Also in this case, if $h_{p}=1$ the initial minimum is lost and the feedback does not provide any noise attenuation.

\section{B. Feedback from the enzyme}

Regards to the feedback from the enzyme, an analytical solution is easier to achieve, since $\left\langle n_{e}^{2}\right\rangle$ and $\left\langle X n_{e}^{2}\right\rangle$ equations 

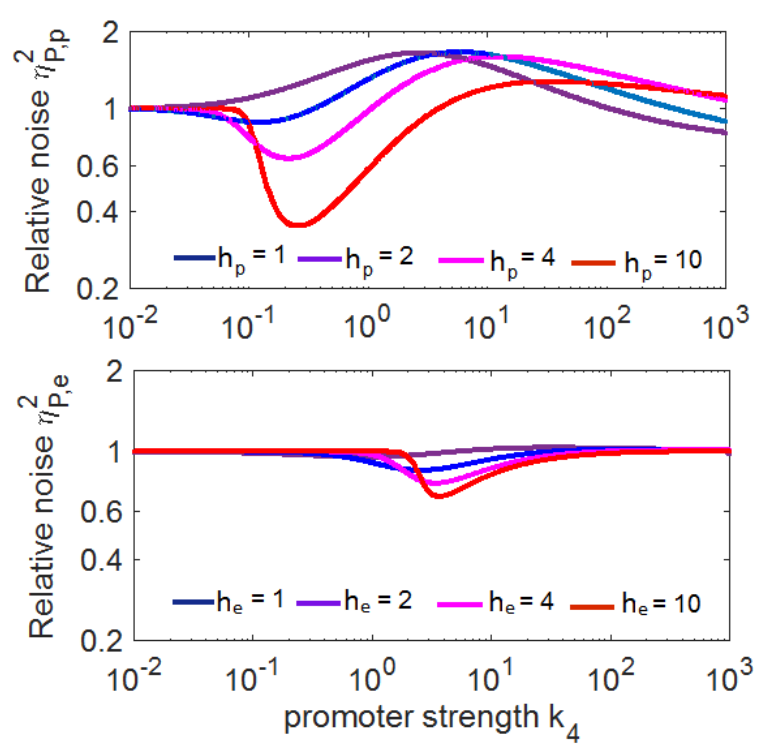

Fig. 3. Relative noise according to varying values of the promoter strength $k_{4}$ : feedback from the product (upper panel, $\theta_{p}=500$ ) and from the enzyme (lower panel, $\theta_{e}=500$ ). $n_{s}=3000$.
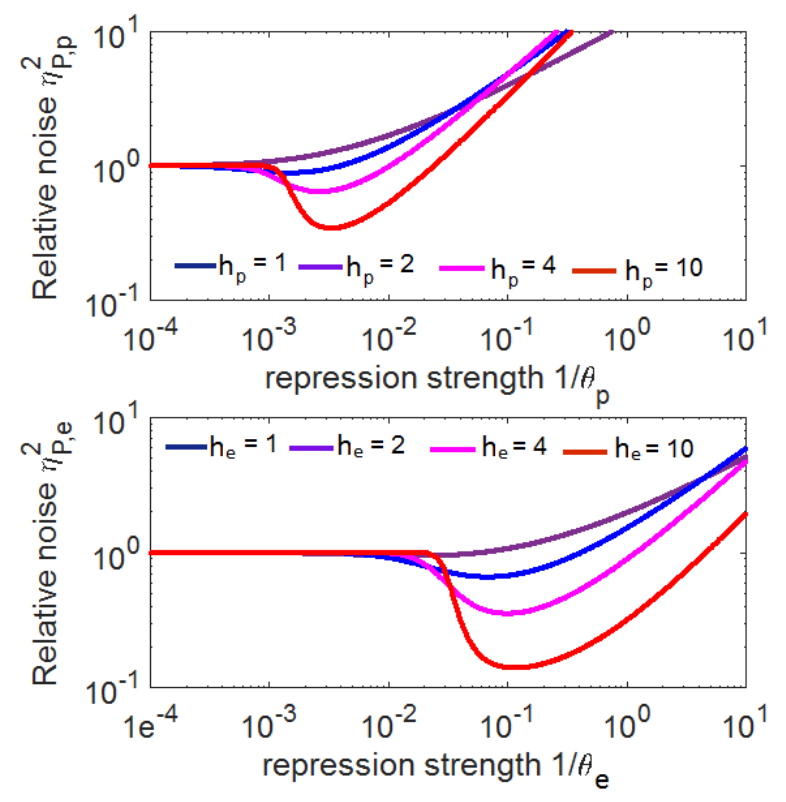

Fig. 4. Relative noise according to varying values of the repression strength $1 / \theta$ : feedback from the product (upper panel) and from the enzyme (lower panel). $k_{4}=0.16, n_{s}=3000$.

are decoupled from the others and provide the following steady-state solutions:

$$
\begin{gathered}
\left\langle n_{e}^{2}\right\rangle^{\star}=\left(n_{e}^{\star}\right)^{2}\left(1+\frac{k_{5}}{2\left\langle B_{e}\right\rangle n_{e}^{\star}\left(k_{5}-\left\langle B_{e}\right\rangle f_{e}^{\prime}\left(n_{e}^{\star}\right)\right)}\left\langle B_{e}^{2}\right\rangle\right) \\
\left\langle X n_{e}^{2}\right\rangle^{\star}=\left\langle n_{e}^{2}\right\rangle^{\star} \cdot X^{\star}
\end{gathered}
$$

Thus, the moments system reduces to a 4-th order system, whose solution for $\left\langle n_{p}^{2}\right\rangle^{\star}$ provides the following metabolic noise:

$$
\eta_{P, e}^{2}=\frac{\Xi_{1}}{\Xi_{2}}+\frac{\Xi_{3}}{\Xi_{4}} \cdot \frac{k_{5} k_{6} X^{\star}}{2\left\langle B_{e}\right\rangle n_{e}^{\star}\left[k_{5}^{F}\left(n_{e}^{\star}\right)\right]^{2}} \cdot\left\langle B_{e}^{2}\right\rangle
$$

where

$$
\begin{gathered}
\Xi_{1}=k_{6} X^{\star}\left(1-X^{\star}\right)\left(k_{8}+k_{6} X^{\star}\left(1+X^{\star}\right)\right) \\
\Xi_{2}=\left(k_{8}+k_{6} X^{\star}\left(1-X^{\star}\right)\right)^{2} \\
\Xi_{3}=3 k_{6} k_{8} X^{\star}\left(1-X^{\star}\right)+k_{5}^{F}\left(n_{e}^{\star}\right) k_{8} X^{\star}+k_{8}^{2} \\
+2 k_{6}\left(X^{\star}\right)^{2}\left(1-X^{\star}\right)\left(k_{5}^{F}\left(n_{e}^{\star}\right)+k_{6}\right) \\
\Xi_{4}=\Xi_{2}\left(1+\frac{k_{6} X^{\star}\left(k_{5}^{F}\left(n_{e}^{\star}\right)+k_{8}\right)}{k_{5}^{F}\left(n_{e}^{\star}\right) k_{8}}+\frac{k_{5}^{F}\left(n_{e}^{\star}\right) X^{\star}}{k_{8}}\right)
\end{gathered}
$$

and

$$
k_{5}^{F}\left(n_{e}^{\star}\right)=k_{5}-\left\langle B_{e}\right\rangle f_{e}^{\prime}\left(n_{e}^{\star}\right) .
$$

What is apparent from (20) is that the two noise sources do not contribute to metabolic noise in a separable fashion. Solutions for varying values of the promoter strength $k_{4}$ and of the repression strength $1 / \theta_{e}$ are reported in the lower panels of Figs. 3 and 4, respectively. There are analogies and differences with the behavior of the other feedback, which are below resumed:

i) like the feedback from the product, by increasing the promoter strength $k_{4}$ we find a minimum of relative noise for promoter sensitivities $h_{e}>1$; however, such a minimum provides a smaller reduction in the relative noise making so preferable the choice of a feedback from the product by using $k_{4}$ as a tuning parameter;

ii) the range of variation of the relative noise is smaller (than the one occurring for the feedback from the product) and occurs on a smaller range of values of $k_{4}$ : again, this makes preferable the use of a feedback from the product, more sensitive to the promoter strength $k_{4}$;

iii) by varying the repression strength $1 / \theta_{e}$ we find a relative noise behavior which is very similar to the analogous case of the feedback from the product; however, the feedback from the enzyme provides a larger range of variation (in terms of reduction) of the relative noise on a larger range of values of the repression strength: in this case, the use of a feedback from the enzyme provides an effect more sensitive to $1 / \theta_{e}$.

\section{The role of the switch $X$}

The frequency and the average steady-state value of the switch $X$ do seem to play an active role in metabolic noise propagation. Indeed, regards to the feedback from the product, by decreasing the value of $k_{8}$ to zero a substantial reduction of the relative noise occurs (Fig.5, upper panel). Instead, by increasing $k_{8}$ we weaken the improvements in metabolic noise reduction. In case of $h_{p}>1$ we can even have a relative noise $>1$. These effects are turned upside down in the feedback from the enzyme (Fig.5, lower panel), since low values of $k_{8}$ make the feedback ineffective $\left(\tilde{\eta}_{P, e}^{2} \simeq 1\right)$, whilst high values of $k_{8}$ provide a reduction in the relative noise, at least for promoter sensitivity $h_{e}>1$. 


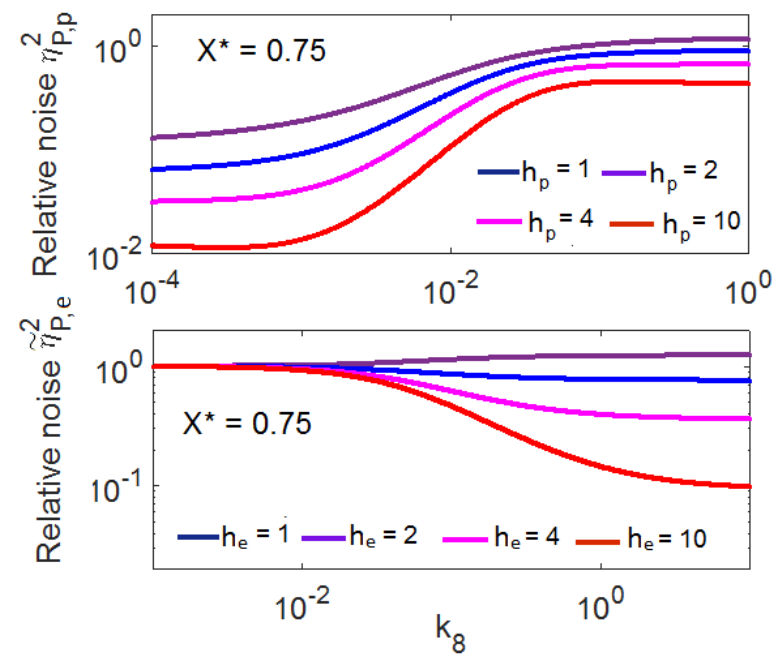

Fig. 5. Relative noise for varying values of $k_{8}$ : feedback from the product (upper panel, $\theta_{p}=500$ ) and from the enzyme (lower panel, $\theta_{e}=5$ ). $k_{4}=0.16, n_{s}=3000$.

\section{Conclusions}

Moment equations have been computed (and steady-state solutions have been discussed) for a basic enzymatic scheme with the aim of investigate the role of feedback in noise reduction. The model accounts for two noise sources: one in the enzyme production, the other in product elimination. The comparison of the solutions for the two different feedbacks suggests the following conclusions. First, by varying the promoter strength $k_{4}$ a minimum of the relative noise is achieved (with $\tilde{\eta}_{P}^{2}<1$ ) in both the feedback schemes, with the scheme involving the feedback from the product to be preferable because more sensitive to $k_{4}$ and providing a better improvement in noise reduction. Second, by varying the repression strength we have a minimum of relative noise as well (with $\tilde{\eta}_{P}^{2}<1$ ) in both feedback schemes, but in this case the scheme with the feedback from the enzyme has to be preferred for a greater sensitivity to $1 / \theta$ and a better improvement in noise reduction. Both feedback schemes share the fact that a greater promoter sensitivity improves the performances in terms of noise reduction. Finally, the frequency and the steady-state value of the stochastic switch that activates/inhibits the product clearance plays an important role in assessing noise reduction. Further investigation is expected to analyze in more details these preliminary results, possibly exploiting the analytical solution provided in this note for the feedback from the enzyme.

\section{ACKNOWLEDGEMENTS}

PP is supported by the MIUR grant SysBioNet Italian Roadmap for ESFRI Research Infrastructures, SYSBIO Centre of Systems Biology, Milan and Rome, Italy. AS is supported by the National Science Foundation Grant DMS1312926.

\section{REFERENCES}

[1] D.E. Cameron et al, A brief history of synthetic biology, Nat. Rev. Microbiol., 12, 381-390, 2014.
[2] D. Del Vecchio, and E. D. Sontag, Synthetic biology: A systems engineering perspective, Control Theory and Systems Biology, 101124, 2009.

[3] D. Del Vecchio, A. J. Ninfa, and E. D. Sontag, Modular cell biology: retroactivity and insulation, Molecular systems biology, 4.1, 161, 2008.

[4] D. Del Vecchio, Modularity, context-dependence, and insulation in engineering biological circuits, Trends in Biotechnology, 33(2), 111$119,2015$.

[5] D.H. Calhoun, G.W. Hatfield, Autoregulation: a role for a biosynthetic enzyme in the control of gene expression, Proceedings of the National Academy of Sciences 70.10 (1973): 2757-2761.

[6] G. Stephanopoulos, A. Aristidou, J. Nielsen, Metabolic Engineering: Principles and Methodologies, Academic Press, San Diego, CA, 1998

[7] A. Zaslaver, A. Mayo, R. Rosenberg, P. Bashkin, H. Sberro, M. Tsalyuk, M. Surette, U. Alon, Just-in-time transcription program in metabolic pathways, Nat. Genet. 36, 486-491, 2004.

[8] U. Alon, An Introduction to Systems Biology: Design Principles of Biological Circuits, Chapman and Hall/CRC, 2006.

[9] A. Singh, J.P. Hespanha, Optimal feedback strength for noise suppression in autoregulatory gene networks, Biophysical Journal 96, 40134023, 2009

[10] W.J. Holtz, J.D. Keasling, Engineering static and dynamic control of synthetic pathways, Cell, 140, 19-23, 2010.

[11] Oyarzun, D.A, Lugagne, J.-B., Stan, G.-B.V.: Noise propagation in synthetic gene circuits for metabolic control, ACS Synthetic Biology, 2014.

[12] A. Borri, P. Palumbo, A. Singh, Metabolic noise reduction for enzymatic reactions: the role of a negative feedback, Proceedings of the 54th IEEE Conference on Decision and Control (CDC 2015), Osaka, Japan, pp. 2537-2542, 2015.

[13] A. Borri, P. Palumbo, A. Singh, Impavct of negative feedback in metabolic noise propagation, IET Syst. Biol., pp. 1-8, 2016.

[14] E.D. Sontag, A. Singh, Exact moment dynamics for feedforward nonlinear chemical reaction networks, IEEE Life Science Letters 1(2), 2015.

[15] M. Soltani, C. Vargas, A. Singh, Conditional moment closure schemes for studying stochastic dynamics of genetic circuits. IEEE Transactions on Biomedical Circuits and Systems, 9(4), 518-526, 2015.

[16] T. Platini, M. Soltani, A. Singh, Stochastic Analysis Of An Incoherent Feedforward Genetic Motif, arXiv:1509.09192 [q-bio.MN]

[17] Segel, L.: On the validity of the steady state assumption of enzyme kinetics. Bull. Math. Biol. 50, 579593 (1988)

[18] A.M. Bersani, E. Bersani, L. Mastroeni, Deterministic and stochastic models of enzymatic networksapplications to pharmaceutical research, Computers and Mathematics with Applications 55, 879-888 (2008).

[19] I. Golding, J. Paulsson, S. Zawilski, E. Cox, Real-time kinetics of gene activity in individual bacteria, Cell 123, 1025-1036, 2005.

[20] J.P. Hespanha, A. Singh, Stochastic models for chemically reacting systems using polynomial stochastic hybrid systems, Int. J. of Robust and Nonlinear Control, 15, 669-689, 2005.

[21] A. Singh, J.P. Hespanha, Approximate moment dynamics for chemically reacting systems, IEEE Transactions on Automatic Control. 56, 414-418, 2011.

[22] D. T. Gillespie, Exact Stochastic Simulation of Coupled Chemical Reactions, The Journal of Physical Chemistry 81(25), 23402361, 1977. 